\title{
Pseudoxanthoma elasticum-like papillary dermal elastolysis in an elderly male
}

\section{Natsumi Norikawa, Yasunobu Kato, Toshiyuki Yamamoto}

\author{
Department of Dermatology, Fukushima Medical University, Fukushima, Japan
}

Corresponding author: Dr. Natsumi Norikawa, E-mail: nnatsumi@fmu.ac.jp

Sir,

Pseudoxanthoma elasticum-like papillary dermal elastolysis (PXE-PDE) clinically presents with whitish, yellow papules on the neck mimicking PXE, but histologically lacks elastic fibers in the papillary dermis [1]. Previous reports have shown that PXE-PDE exclusively involves elderly women. We herein report a rare male case of pseudoxanthoma elasticum-like papillary dermal elastolysis.

An 84-year-old man visited our department, complaining of a number of asymptomatic tiny papules on the neck, which he noticed lyear previously. He had been working as a farmer, but denied any trauma of triggering events on that site. He was taking antihypertensive drugs but otherwise healthy. Physical examination showed a number of yellowish tiny papules on the bilateral neck (Figs. la and $1 \mathrm{~b}$ ). A number of longitudinal wrinkles were observed in the area between nose and upper lip. Histological examination revealed almost normal epidermis, intense solar elastosis and many dilated blood vessels in the upper dermis (Fig. 2a). Other intriginous areas such as axilla and groins were uninvolved. Solar elastosis was positively stained by Weigert staining and Elastica van Gieson staining (Figs. 2b and 2c). Kossa staining did not reveal calcified materials in the dermis. Unfortunately, there are no satisfactory remedies, and the patient did not want surgical removal of the remaining lesion.

The present case developed a number of small slightly yellowish papules disseminated in the bilateral neck. PXE-PDE mostly affects elderly females (in the seventh decade) and presents with multiple yellowish non-follicular cobblestone-like papules which may

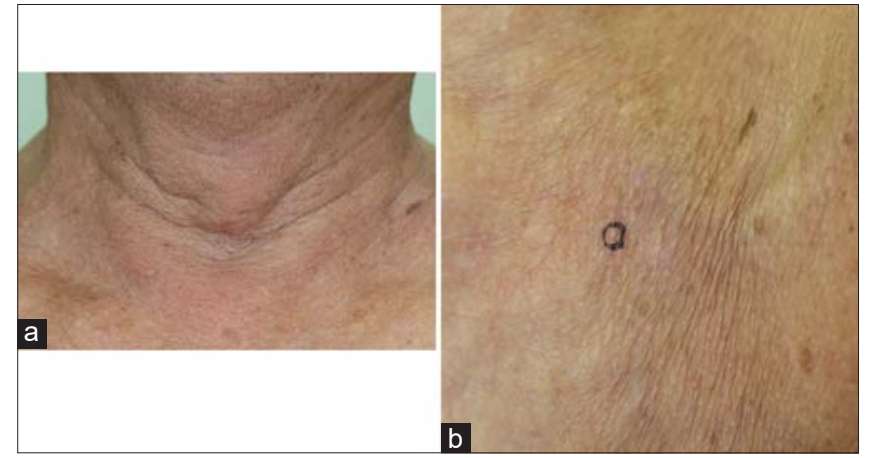

Figure 1: $(a$ and $b)$ A number of yellowish tiny papules on the bilateral neck were observed.

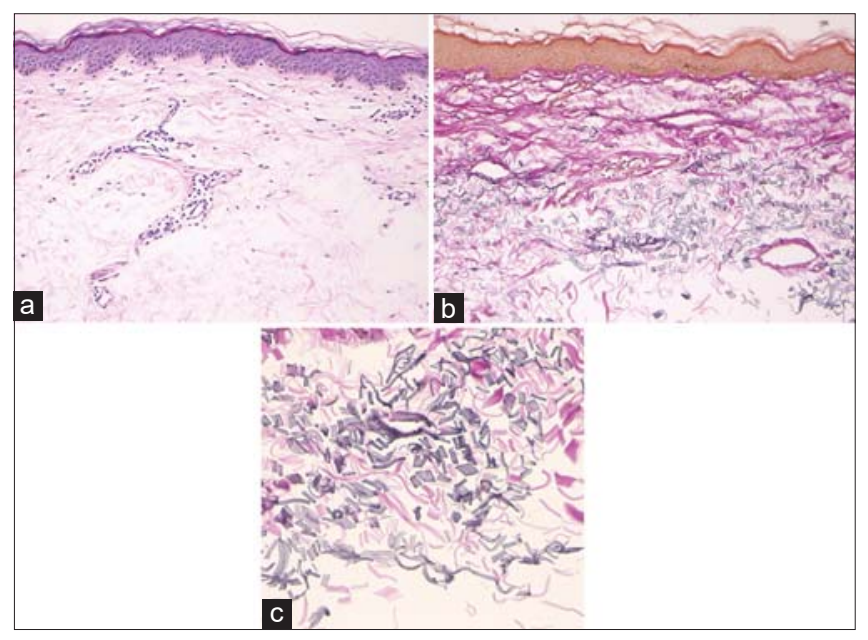

Figure 2: (a) Histological features showed almost normal epidermis, intense solar elastosis and many dilated blood vessels in the upper dermis (Hematoxylin and eosin stain, original magnification $\times 100$ ). (b and c) Disruption of elastic fibers in the mid-dermis (Elastica van Gieson staining, original magnification: $b \times 100, c \times 400)$.

coalesce into plaques on the neck, flexor forearms, and axillae [1]. Histological features show a marked loss of elastic fibers in the papillary dermis. In addition,

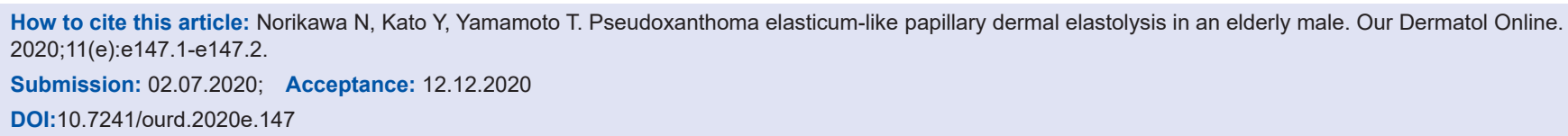


elastic fibers in the mid-dermis were shortened. Kossa stain did not show calcification [2]. Fragmentation of elastic fibers is usually absent, whereas rarely observed in PXE-PDE, suggesting that elastolytic change can be seen not only in the papillary dermis but also in the subpapillary dermis.

The primary pathologic condition of PXE-PDE may be elastolytic change, possibly caused by ultraviolet radiation damage, intrinsic aging, genetic or inheritable factors, and abnormal elastogenesis. Male occurrence is few with only a few reports [3], which however may be underreported. There are several disorders that should be differentiated from PXE-PDE. Late-onset focal dermal elastosis clinically resembles PXE-PDE; which, however, is easily differentiated by their histological features. Mid-dermal elastolysis exhibits clinically well-demarcated wrinkling and perifollicular papules with 'peau d'orange' appearance, and is characterized by band-like focal loss of elastic fibers in the middermis [4]. Mid-dermal elastosis frequently affects the trunk and upper proximal extremities, and has been associated with different autoimmune diseases. Anetoderma usually involves the trunk and proximal extremities, and histologically shows loss of elastic fibers in the papillary and reticular dermis. Cutis punctata linearis colli is a disease showing similar clinical symptoms. This is the presence on the V-area of the neck and yellowish, closely set maculopapules arranged in a delicate tracery of short or long undulating lines. However, histopathology showed the sebaceous glands were more numerous and larger, associated with hair follicles.

\section{Consent}

The examination of the patient was conducted according to the principles of the Declaration of Helsinki.

The authors certify that they have obtained all appropriate patient consent forms, in which the patients gave their consent for images and other clinical information to be included in the journal. The patients understand that their names and initials will not be published and due effort will be made to conceal their identity, but that anonymity cannot be guaranteed.

\section{REFERENCES}

1. Aljoudi SB, Abdujabbar MH, Hariri JO. A case series of pseudoxanthoma elasticum-like disorders. Indian J Dermatol. 2019;64:482-5.

2. Rebeiro CP, Abuawad YG, Swickzar BCC, Valente NYS. Pseudoxanthoma elasticum-like papillary dermal elastolysis. An Bras Dermatol. 2017;92:897-8.

3. Panagou E, Ratynska M, Heelan K. Pseudoxanthoma elasticum-like papillary dermal elastolysis: a case report and review of literature. Int J Dermatol. 2019;58:93-7.

4. Alves R, Ferreira L, Vale E, Bordalo O. Pseudoxanthoma elasticum papillary dermal elastolysis: a case report. Dermatol Res Pract. 2010;2010.

Copyright by Natsumi Norikawa, et al. This is an open access article distributed under the terms of the Creative Commons Attribution License, which permits unrestricted use, distribution, and reproduction in any medium, provided the original author and source are credited.

Source of Support: Nil, Conflict of Interest: None declared. 